Research Article

\title{
Dynamic Modeling and CAE Cosimulation Method for Heavy-Duty Concrete Spreader
}

\author{
Shiying Zhang, ${ }^{1,2}$ Ke Zhang, ${ }^{1}$ Bo Song, ${ }^{3}$ Wenda $\mathrm{Yu},{ }^{1}$ and Dong Li $\mathbb{C D}^{1}$ \\ ${ }^{1}$ School of Mechanical Engineering, Shenyang Jianzhu University, Shenyang, China \\ ${ }^{2}$ Northern Heavy Industries Group Co., Ltd., Shenyang, Liaoning, China \\ ${ }^{3}$ CCTEG Shenyang Engineering Company, Shenyang, Liaoning, China \\ Correspondence should be addressed to Dong Li; lidong@sjzu.edu.cn
}

Received 2 February 2021; Revised 26 February 2021; Accepted 17 March 2021; Published 30 March 2021

Academic Editor: Sang-Bing Tsai

Copyright (c) 2021 Shiying Zhang et al. This is an open access article distributed under the Creative Commons Attribution License, which permits unrestricted use, distribution, and reproduction in any medium, provided the original work is properly cited.

This paper presents the dynamic model of heavy-duty concrete spreader with liquid-solid rigid-flexible coupling by means of mathematical modeling and CAE cosimulation. The mathematical method of liquid-solid dynamic model of heavy-duty concrete spreader is described. Based on the liquid-solid coupling system, two degrees of freedom are added to change the model into a liquid-solid rigid-flexible coupling model, and the calculation process of the model is given in detail. The results show that, considering two flexible body factors, the solution scale is relatively large and the complexity of mathematical model derivation is increased. It is very difficult to establish a general dynamic equation which can be easily solved by computer. Therefore, this paper presents a new method of CAE cosimulation of liquid-solid rigid-flexible coupling. This method is divided into two parts: the computer simulation process of liquid-solid coupling and the computer simulation process of rigid-flexible coupling. First, the fluid-solid coupling is carried out by COMSOL software, and then the rigid-flexible coupling is carried out by HyperMesh software, Ansys software, and Adams software. This method can easily establish the dynamic model of the liquid-solid rigidflexible coupling system, which provides a new idea for the simulation of heavy-duty concrete spreader. The simulation results can provide valuable insights into product design and structural optimization.

\section{Introduction}

In recent years, precast concrete members have been developing rapidly and the heavy-duty concrete spreader is an important equipment in the production line of precast concrete components. The performance of the concrete spreader will affect the efficiency of the whole production line. Multispiral heavy-duty concrete spreader has the advantages of strong adaptability, good operability, high spreading efficiency, and advanced automation performance [1-4].

At present, there are few researches on the dynamics of heavy-duty concrete spreader. In contrast, the similar dynamic characteristics of cranes are studied. For example, Japanese scholar used the Newton-Raphson iterative method to establish a velocity model to study vibration effects [5]. American scholar developed a systematic fuzzy controller to achieve the swing of the wire rope during crane operation [6]. Sun of Northeastern University conducted a stability study on the vibration law of the crawler crane system based on virtual prototype technology [7]. Wang et al. of Nankai University established the dynamic mathematical model of the rotary jib type marine crane by using the Lagrangian analysis method and verified the accuracy of the model by simulation test [8].

Modeling the system of heavy-duty concrete spreader is an important means to study its performance. Because of the different methods of analysis, there are various methods of system modeling. Newton's motion theorem and Lagrange's dynamics theory are the most commonly used systems modeling theories. Newtonian mechanics mainly focus on the force analysis of a single particle, while the concrete spreader system is relatively complex, so it is difficult to establish an accurate mathematical model with Newton's 
motion theorem. Lagrange's equation is based on the analysis of the energy in the working process of the heavy-duty concrete spreader. Considering the whole kinetic energy and gravity potential energy of the system, by establishing the energy function of the system, the nonbinding dynamic equation is established for the horizontal and vertical motion of the spreader. The system model established by EulerLagrange equation fully reflects the dynamic structure of the heavy-duty concrete spreader, which is simpler than Newton's motion theorem, and lays a foundation for further research on the performance and control of the heavy-duty concrete spreader. But, for the heavy-duty concrete spreader system, the dynamic model established by Euler-Lagrange equation is also very complex.

In recent years, in the field of mechanical manufacturing, the maturity and perfection of CAE simulation technology have led the development of modern manufacturing technology to a higher level. The development of digital development in virtual environment can not only optimize the design of structure but also complete simulation experiments $[9,10]$. It even replaces experiments that are difficult to be accomplished through physical experiments and realizes the virtual manufacturing of mechanical products [11-13]. The model of the product is built by CAD/CAE simulation, and the simulation analysis is carried out for various working conditions before it is put into use, so as to improve the design of the product and improve the performance of the product.

\section{The Structure Characteristics and Working Principle}

The main function of concrete spreader is to homogenize the concrete through the dispersing device and uniformly output the concrete into the mold [14-16]. The structure is shown in Figure 1.

The cart is driven by two motors by gear rack device and can walk along the frame in the $Y$ direction, and the parallel track on the cart serves as the route for the trolley. The walking trolley is driven by a motor by gear rack device along the track in the $X$ direction. The dispersing device is used to carry concrete and implement the spreading process. It is mainly composed of a funnel-type feeder, a casing, a concrete conveying device, a scatter device, a pneumatic gate, a bottom plate, and a weighting sensor, as shown in Figure 2.

According to the spreading mode, the concrete conveying device can be divided into spiral conveying and spider shaft conveying. The working process of the spreader is as follows: the lubrication device is enabled before starting the concrete spreader to ensure that the oil can continuously lubricate the spiral shaft. Then, the concrete feeder is moved to the feeding position to receive the concrete. The cart and trolley are moved to the desired spreading area using remote controller. Open the pneumatic gates, the number of which is calculated according to the required spreading width. At the same time, the conveying motors are started, and the concrete is slowly pushed out of the dispersing device by the spirals. The cart and trolley are moved again to complete the spreading work in all areas. When all the precast

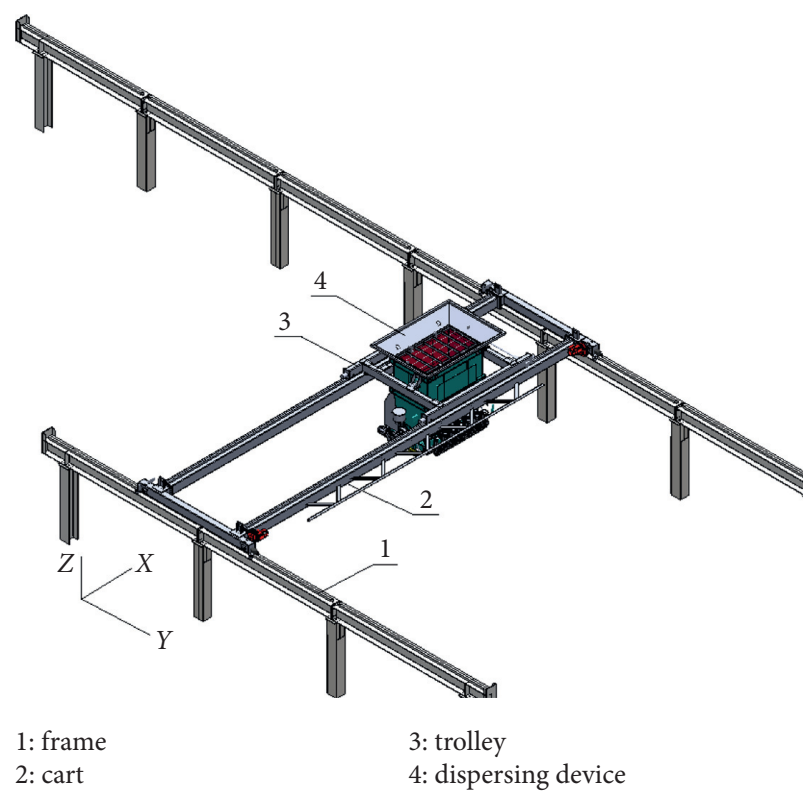

Figure 1: The structure of concrete spreader.

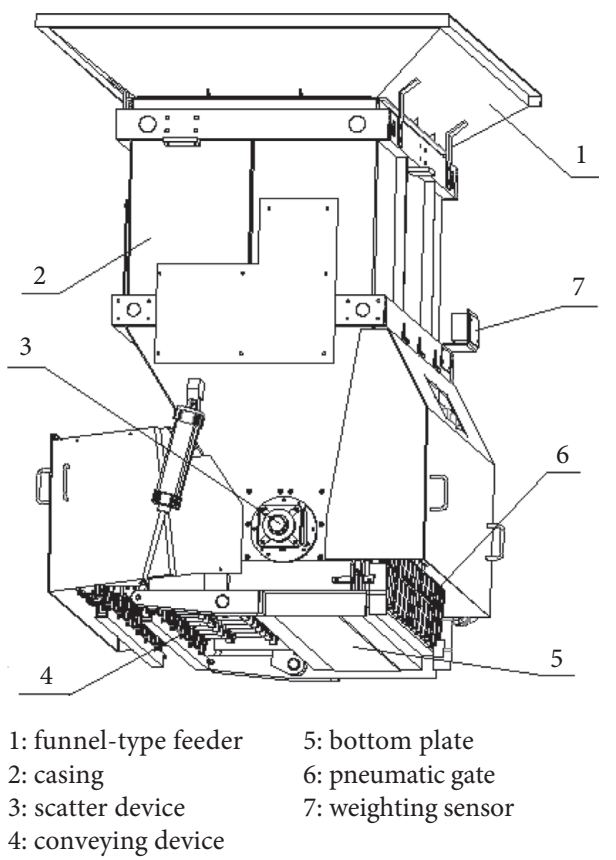

FIgURe 2: The dispersing device.

components are completed, the concrete spreader will be moved to the cleaning position, and then all the gates and the bottom plate are opened, and the residual concrete inside the spreader is cleaned with a high-pressure water gun for the next round of work [17-19].

It can be seen that the concrete spreader system is a nonlinear underactuated system with fewer input variables than output variables. In addition, the complexity of its working environment, as well as the influence of uncertain factors such as external wind, air resistance, and friction resistance between the trolley and the guide rail, makes the 
trolley show serious time-varying and nonlinear characteristics in the working process. Therefore, it is not easy to build an accurate model. For the convenience of modeling, the following assumptions and requirements are given: The quality of the cart is much smaller than that of the trolley, dispersing device, and load, which can be neglected. Cart and loaded dispersing device can be treated as particles when modeling. The friction of the connecting parts of the concrete spreader is neglected.

\section{Dynamic Model of Heavy-Duty Concrete Spreader}

The Lagrangian method provides a powerful analysis tool for multivariable, multiconstrained, strongly coupled dynamic systems.

\section{Modeling Method of Dynamic Model for Liquid-Solid Multibody System of Spreader}

Figure 3 is the system model of the concrete spreader, wherein $x_{M}, y_{M}$, and $z_{M}$ represent the centroid coordinates of the trolley, and $x_{m}, y_{m}$, and $z_{m}$ represent the centroid coordinates of the loaded dispersing device. The load in the spreader is concrete. During the movement of the trolley, the concrete will sway, which will cause the change of the centroid position of the loaded dispersing device. $M$ denotes the mass of the trolley, $m$ denotes the mass of the loaded dispersing device, and $l$ denotes the distance between the centroid coordinates of the loaded dispersing device and the centroid coordinates of the trolley. $\theta_{x}$ denotes the angle between the $Z$-axis and the line that is projected on the $X Z$ plane through connecting the center of mass of the loaded dispersing device and the center of mass of the trolley. $\theta_{y}$ denotes the angle between the line connecting the center of mass of the loaded dispersing device and the center of mass of the trolley and the $X Z$ plane. $f_{x}$ and $f_{y}$ are the horizontal driving forces of $X$-axis and $Y$-axis, respectively. follows.

The process of establishing the dynamic model is as

The trolley's centroid coordinates can be expressed by the following formula:

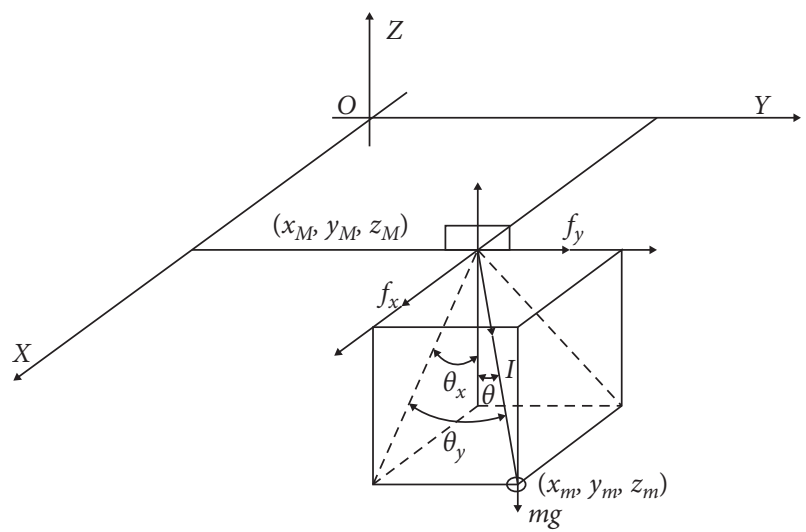

FIgURE 3: The system model of the concrete spreader.

$$
\left\{\begin{array}{l}
x_{M}=x \\
y_{M}=y \\
z_{M}=0
\end{array}\right.
$$

Similarly, the centroid coordinates of the loaded dispersing device can be expressed as

$$
\left\{\begin{array}{l}
x_{m}=x+l \sin \theta_{x} \cos \theta_{y} \\
y_{m}=y+l \sin \theta_{y} \\
z_{m}=-l \cos \theta_{x} \cos \theta_{y} .
\end{array}\right.
$$

The velocity components of the trolley on the axle of $X$, $Y$, and $Z$ can be expressed as follows by derivation of formula (1):

$$
\left\{\begin{array}{l}
\dot{x}_{M}=\dot{x} \\
\dot{y}_{M}=\dot{y} \\
\dot{z}_{M}=0
\end{array}\right.
$$

Similarly, the velocity components of the loaded dispersing device on $X$-axis, $Y$-axis, and $Z$-axis can be expressed as follows:

$$
\left\{\begin{array}{l}
\dot{x}_{m}=\dot{x}+i \sin \theta_{x} \cos \theta_{y}+\dot{\theta}_{x} \cos \theta_{x} \cos \theta_{y}-i \dot{\theta}_{y} \sin \theta_{x} \sin \theta_{x} \sin \theta_{y}, \\
\dot{y}_{m}=\dot{y}+i \sin \theta_{y}+\dot{\theta}_{y} \cos \theta_{y} \\
\dot{z}_{m}=-i \cos \theta_{x} \cos \theta_{y}+l \dot{\theta}_{x} \sin \theta_{x} \cos \theta_{y}+l \dot{\theta}_{y} \cos \theta_{x} \sin \theta_{y} .
\end{array}\right.
$$

Then the kinetic energy of the system is the sum of the

$$
\begin{aligned}
T= & \frac{1}{2} M v_{M}^{2}+\frac{1}{2} m v_{m}^{2}=\frac{1}{2} M\left(\dot{x}_{M}^{2}+\dot{y}_{m}^{2}+\dot{z}_{M}^{2}\right) \\
& +\frac{1}{2} m\left(\dot{x}_{M}^{2}+\dot{y}_{m}^{2}+\dot{z}_{M}^{2}\right) .
\end{aligned}
$$


The lowest point of centroid coordinates during the operation of the loaded dispersing device is used as the relative zero potential reference point of the system. The potential energy of the system can be expressed as follows:

$$
V=m g l-m g l \cos \theta_{x} \cos \theta_{y} .
$$

Then the Lagrangian function of the system is

$$
\begin{aligned}
L=T-V \frac{1}{2} M\left(\dot{x}^{2}+\dot{y}^{2}\right)+\frac{1}{2} m\left(\begin{array}{c}
\dot{x}^{2}+\dot{y}^{2}+\dot{l}^{2}-2 \dot{x} \dot{\theta}_{y} l \sin \theta_{x} \sin \theta_{y}+ \\
2 \dot{x} \dot{\theta}_{x} l \cos \theta_{x} \cos \theta_{y} 2 \dot{x} \dot{l}_{\sin } \theta_{x} \cos \theta_{y}+2 \dot{y} l \sin \dot{\theta}_{y}+2 l \dot{y} \dot{\theta}_{y} \cos \theta_{y}+ \\
\dot{\theta}_{x}^{2} l^{2} \cos ^{2} \theta_{y}+\dot{\theta}_{y}^{2} l^{2}
\end{array}\right) \\
+\operatorname{mgl}\left(\cos \theta_{x} \cos \theta_{y}-1\right) .
\end{aligned}
$$

Let the generalized coordinates of the system be $q=\left(x, y, l, \theta_{x}, \theta_{y}\right)^{T}$. From the standard Euler-Lagrange equation of mechanical system:

$$
\frac{\mathrm{d}}{\mathrm{d} t}\left(\frac{\partial L(q, \dot{q})}{\partial \dot{q}}\right)-\left(\frac{\partial L(q, \dot{q})}{\partial q}\right)=Q_{j} \quad(j=1,2, \ldots k)
$$

The dynamic model of the moving mechanism of the heavy-duty concrete spreader is obtained as follows:

$$
\left\{\begin{array}{l}
(M+m) \ddot{x}+m l \cos \theta_{x} \cos \theta_{y} \ddot{\theta}_{x}-m l \sin \theta_{x} \sin \theta_{y} \ddot{\theta}_{y}+m \sin \theta_{x} \cos \theta_{y} \ddot{I}+ \\
B_{x} \dot{x}+2 m \cos \theta_{x} \cos \theta_{y} \ddot{\theta}_{x}-2 m \sin \theta_{x} \sin \theta_{y} \ddot{\theta}_{y}-m l \sin \theta_{x} \cos \theta_{y} \dot{\theta}_{x}^{2}- \\
2 m l \cos \theta_{x} \sin \theta_{y} \dot{\theta}_{x} \dot{\theta}_{y}-m l \sin \theta_{x} \cos \theta_{y} \dot{\theta}_{y}^{2}=f_{x}, \\
(M+m) \ddot{y}+m l \cos \theta_{y} \ddot{\theta}_{y}+m \sin \theta_{y} \ddot{I}+B_{y} \dot{y}+2 m \cos \theta_{y} \ddot{\theta}_{y}-m l \sin \theta_{y} \dot{\theta}_{y}^{2}=f_{y} \\
m \ddot{I}+m \sin \theta_{x} \cos \theta_{y} \ddot{x}+m \sin \theta_{y} \ddot{y}+B_{l} \dot{l}-m l \cos ^{2} \theta_{y} \dot{\theta}_{x}^{2}-m g \cos \theta_{x} \cos \theta_{y}-m l \dot{\theta}_{y}^{2}=0 \\
m l^{2} \cos ^{2} \theta_{y} \ddot{\theta}_{x}+m l \cos \theta_{x} \cos \theta_{y} \ddot{x}-2 m l^{2} \sin \theta_{y} \cos \theta_{y} \dot{\theta}_{x} \dot{\theta}_{y}+2 m l \cos ^{2} \theta_{y} \ddot{\theta}_{x}+m g l \sin \theta_{x} \cos \theta_{y}=0 \\
m l^{2} \ddot{\theta}_{y}+m l \cos \theta_{y} \ddot{y}-m l \sin \theta_{x} \sin \theta_{y} \ddot{x}+m l^{2} \cos \theta_{y} \sin \theta_{y} \dot{\theta}_{x}^{2}+2 m l i \dot{\theta}_{y}+ \\
m g l \cos \theta_{x} \sin \theta_{y}=0 .
\end{array}\right.
$$

\section{Modeling Method of Dynamic Model for Liquid-Solid Rigid-Flexible Multibody System of Spreader}

When considering the beams of the cart as a flexible body, it can be simplified to add two degrees of freedom to the generalized coordinates of rigid system. They are the displacement amount $z_{M}$ of the trolley in the vertical direction and the rotation angle $\theta_{M}$ of the trolley along the $X$-axis. $r$ is the length of the beam of the trolley. $k$ is the stiffness coefficient of the beam of the cart, and $k$ is a variable whose magnitude is related to the position of the flexible beam. $J$ is the moment of inertia along the $X$-axis of the cart and the loaded dispersing device. The system dynamics model is established as follows.
The trolley's centroid coordinates can be expressed by the following formula:

$$
\left\{\begin{array}{l}
x_{M}=x, \\
y_{M}=y, \\
z_{M}=z, \\
\theta_{M}=0 .
\end{array}\right.
$$

Similarly, the centroid coordinates of the loaded dispersing device can be expressed as

$$
\left\{\begin{array}{l}
x_{M}=x+l \sin \theta_{y} \cos \theta_{y} \\
y_{M}=y+l \sin \theta_{y} \\
z_{M}=z-l \cos \theta_{x} \cos \theta_{y}
\end{array}\right.
$$


The velocity components of the trolley on the axle of $X$, $Y$, and $Z$ can be expressed as follows by derivation of formula (10):

$$
\left\{\begin{array}{l}
\dot{x}_{M}=\dot{x}, \\
\dot{y}_{M}=\dot{y} \\
\dot{z}_{M}=\dot{z}, \\
\dot{\theta}_{M}=\dot{\theta} .
\end{array}\right.
$$

Similarly, the velocity components of the loaded dispersing device on $X$-axis, $Y$-axis, and $Z$-axis can be expressed as follows:

$$
\left\{\begin{array}{l}
\dot{x}_{m}=\dot{x}+l \sin \theta_{x} \cos \theta_{y}+l \theta_{x} \cos \theta_{x} \cos \theta_{y}-l \theta_{y} \sin \theta_{x} \sin \theta_{y} \\
\dot{y}_{m}=\dot{y}+i \sin \theta_{y}+i \dot{\theta}_{y} \cos \theta_{y}, \\
\dot{z}_{m}=\dot{z}-i \cos \theta_{x} \cos \theta_{y}+i \dot{\theta}_{x} \sin \theta_{x} \cos \theta_{y}+l \dot{\theta}_{y} \cos \theta_{x} \sin \theta_{y} .
\end{array}\right.
$$

Then, the kinetic energy of the system is the sum of the kinetic energy of the trolley and the kinetic energy of the loaded dispersing device:

$$
\begin{aligned}
T= & \frac{1}{2} M v_{M}^{2}+\frac{1}{2} J\left(\dot{\theta}_{M}\right)^{2}+\frac{1}{2} m v_{m}^{2}=\frac{1}{2} M\left\{(\dot{x})^{2}+(\dot{y})^{2}+(\dot{z})^{2}\right\}+ \\
& \frac{1}{2} m\left(\dot{x}+i \sin \theta_{x} \cos \theta_{y}+\dot{\theta}_{x} \cos \theta_{x} \cos \theta_{y}-i \dot{\theta}_{y} \sin \theta_{x} \sin \theta_{y}\right)^{2}+ \\
& \frac{1}{2} m\left(\dot{y}+i \sin \theta_{y}+i \dot{\theta}_{y} \cos \theta_{y}\right)^{2}+\frac{1}{2} m\left(\begin{array}{c}
\dot{z}-i \cos \theta_{x} \cos \theta_{y}+ \\
i \dot{\theta}_{x} \sin \theta_{x} \sin \theta_{y}+l \dot{\theta}_{y} \cos \theta_{x} \sin \theta_{y}
\end{array}\right)^{2}+\frac{1}{2} J(\dot{\theta}) .
\end{aligned}
$$
follows:

The potential energy of the system can be expressed as

$$
V=m g l+\frac{1}{2} k z^{2}+(M+m) g z-m g l \cos \theta_{x} \theta_{y},
$$

where $k$ is the stiffness coefficient of the flexible beam, and the expression is

$$
k=\frac{3 E I r}{x^{2}(r-x)^{2}}
$$

The potential energy of the system is obtained by introducing (16) into (15):

$$
V=m g l+\frac{3 E I r z^{2}}{2 x^{2}(r-x)^{2}}+(M+m) g z-m g l \cos \theta_{x} \cos \theta_{y} .
$$

$$
\begin{aligned}
L= & T-V=\frac{1}{2} M\left\{(\dot{x})^{2}+(\dot{y})^{2}+(\dot{z})^{2}\right\}+\frac{1}{2} m\left(\begin{array}{c}
\dot{x}+i \sin \theta_{x} \cos \theta_{y}+l \dot{\theta}_{x} \cos \theta_{x} \cos \theta_{y}- \\
i \dot{\theta}_{y} \sin \theta_{x} \sin \theta_{y}
\end{array}\right)^{2}+ \\
& \cdot \frac{1}{2} m\left(\dot{y}+i \sin \theta_{y}+i_{\operatorname{lin}} \theta_{y}+i \dot{\theta}_{y} \cos \theta_{y}\right)^{2}+\frac{1}{2} m\left(\dot{z}-i \cos \theta_{x} \cos \theta_{y}+l \dot{\theta}_{x} \sin \theta_{x} \sin \theta_{y}+i \dot{\theta}_{y} \cos \theta_{x} \sin \theta_{y}\right)^{2} \frac{1}{2} J(\dot{\theta})- \\
& \cdot m g l+\frac{1}{2} \frac{3 E I r}{x^{2}(r-x)^{2}} z^{2}+(M+m) g z-m g l \cos \theta_{x} \cos \theta_{y} .
\end{aligned}
$$


Let the generalized coordinates of the system with two degrees of freedom be added. From the standard EulerLagrange equation of mechanical system of formula (8), the dynamic model of liquid-solid rigid-flexible coupling multibody system of heavy-duty concrete spreader can be obtained. From the Lagrange function (18), the solution is very complicated. Therefore, it is not listed here.

It can be seen from the process of establishing the dynamic model that, due to the consideration of two soft body factors, a coupling motion occurs between the rigid motion and the elastic deformation of the flexible body. It increases the complexity of mathematical model derivation, and the solution scale is relatively large. It is very difficult to establish a more versatile equation of motion with more degrees of freedom and computer-solving.

In this paper, a computer simulation method of liquidsolid rigid-flexible coupling loaded concrete spreader is presented, which combines several kinds of software. It makes dynamics simulation very convenient and plays an important role in the research. The simulation results can provide reference for product design and structural optimization.

\section{CAE Cosimulation of Liquid-Solid Rigid- Flexible Multibody System}

In order to accurately obtain the dynamic model of heavyduty concrete spreader, it is necessary to consider the liquidsolid coupling between concrete and dispersing device motion and the rigid-flexible coupling between the deformation of the flexible part of the beam and the rigid body of other parts. Therefore, the whole system is a liquid-solid rigid-flexible coupling system. The method is to first use SolidWorks software to model, use COMSOL software for liquid-solid coupling, and then use HyperMesh software, Ansys software, and Adams software for rigid-flexible coupling. The specific process is shown in Figure 4.

The comprehensive application of CAD/CAE software aims at giving full play to the advantages of each software and avoiding its shortcomings. Thus, the whole process of software geometry modeling, meshing, analysis, and result processing is high-speed, efficient, clear, error-prone, and easy to operate and modify. SolidWorks software, COMSOL software, HyperMesh software, Ansys software, and Adams software have good integration effects, making data interaction more convenient and accurate, so they were chosen. Applying them to the design process of the machine can quickly and intuitively and accurately discover the problems in the design and optimize the design structure. It can be seen that data interaction plays an important role in the comprehensive application of software.

\section{Liquid-Solid Coupling Process}

At present, the study of liquid-solid coupling is important and universal [20, 21]. COMSOL Multiphysics coupling simulation software based on differential control equation can simulate the movement of the free surface of the concrete inside the machine during the movement [22], and through the visualization of the postprocessing results, the crest and trough of liquid and the pressure distribution in the fluid are faithfully reflected. The specific process is as follows.

Modeling: Model the spreader using SolidWorks software and import it into COMSOL software. It should be noted that the imported model cannot be directly analyzed, and the model needs to be modified and simplified [23, 24].

Boundary conditions: add gravity, reference pressure, and pressure constraint points, and set the initial liquid level and open boundary, and the other boundaries are set to fixed wall surfaces.

Initial conditions: the material library is used to give physical parameters corresponding to air and concrete. The density of the concrete is $2400 \mathrm{~kg} / \mathrm{m}^{3}$, and the viscosity of the concrete is $20 \mathrm{P} \cdot \mathrm{s}$. The viscosity can be obtained by the L-box experiment and the slumping area test method, as shown in Figures 5 and 6.

Assuming that the trolley moves in the direction of $X$, the fluid in the dispersing device moves with the dispersing device. Because of inertia, the liquid level of fluid will be left low and right high. It corresponds to the velocity of the fluid in the opposite direction to the movement of the dispersing device. Based on this relative simulation method, the initial velocity in the $X$ direction of the concrete is $-0.4 \mathrm{~m} / \mathrm{s}$, which is used to simulate the motion of the dispersing device.

Meshing: COMSOL comes with a meshing tool. This model uses a free triangle mesh. In order to better simulate the free surface change, select the refinement function, click on the initial liquid level boundary, and change the number of refinements to 3 .

Results and treatment: Figure 7 shows the distribution of the fluid in motion. It can be seen that assigning a negative $X$ direction velocity to the fluid, that is, the velocity of the dispersing device moving to the left, which will cause the phenomenon of low left and high right on the free liquid surface due to inertia, and the fluid will fluctuate in the domain, over time, this fluctuation will gradually weaken. It is found that when $t=0.5 \mathrm{~s}$, the fluctuation of liquid level reaches its maximum value, and when $t=2.5 \mathrm{~s}$, the fluctuation of liquid level tends to flatten obviously.

To determine the maximum value of the peak, an observation section that is parallel to the $X Z$ plane can be added to the model. As shown in Figure 8, the distance between the peak and the initial liquid level can be calculated.

Using COMSOL software, the pressure contours at different time can be obtained, and the change with the liquid level can be observed. The pressure distribution can be loaded into Adams software for dynamic simulation analysis.

\section{Rigid-Flexible Coupling Process}

The principle of establishing rigid-flexible coupling model is to generate flexible parts for slender members with small stiffness, while, for parts with larger stiffness, rigid body model is used [25]. Therefore, the rigid-flexible coupled multibody dynamic model analyzed in this paper only deals with two beams of the cart, while the other parts are rigid 


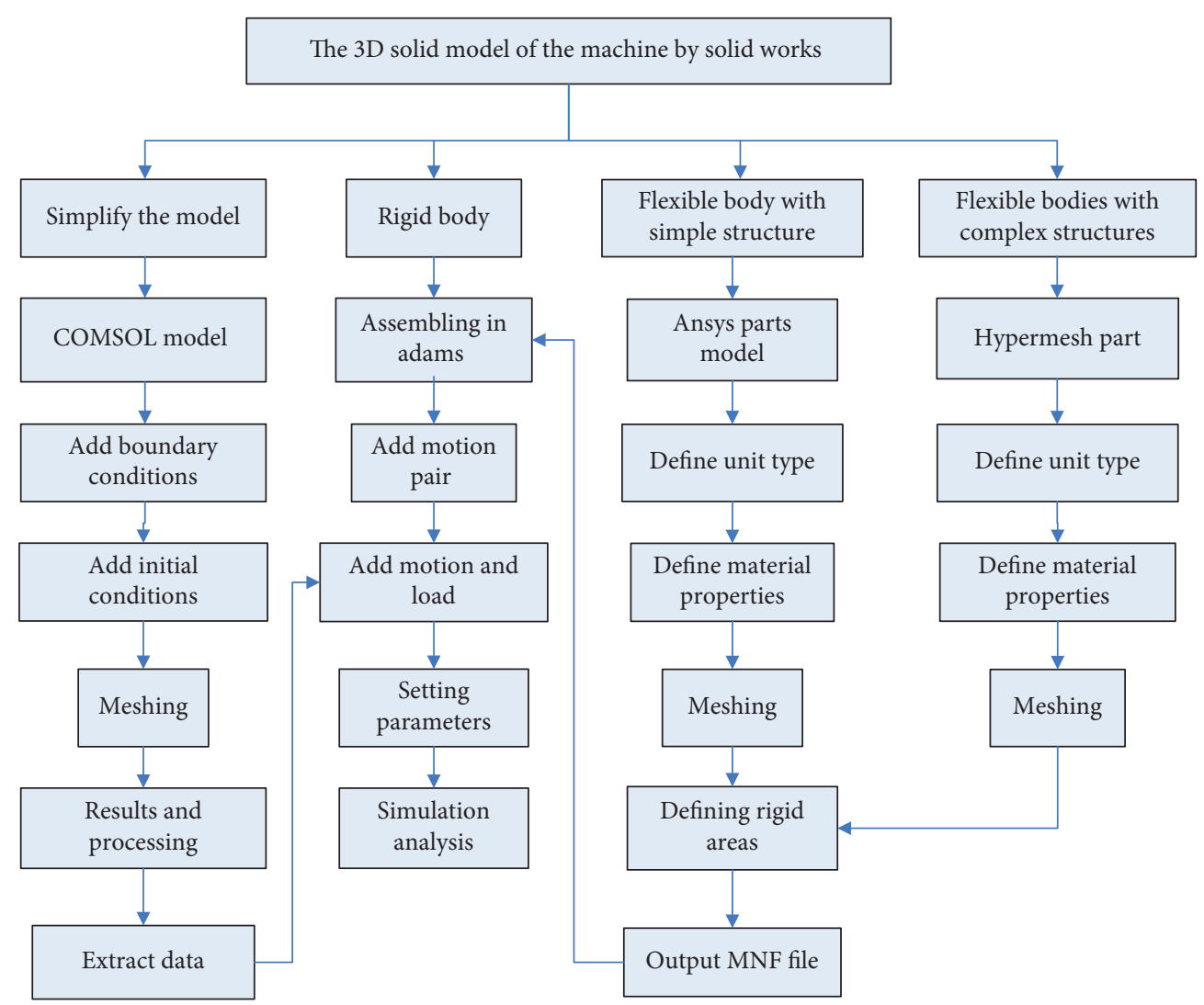

FIgURE 4: CAE cosimulation flow chart of liquid-solid rigid-flexible coupled multibody system.

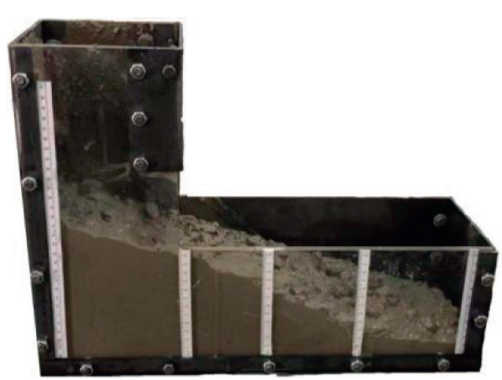

Figure 5: L-box experiment.

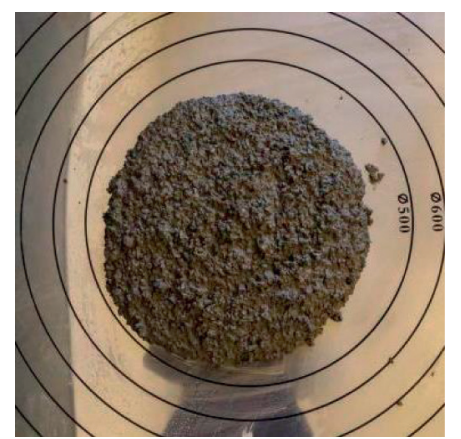

Figure 6: Slumping area experiment.

bodies, as shown in Figure 9. In addition, the elastic deformation of slender body has a significant impact on the output response of the system. If it is simplified as a rigid body, the actual result of parameter identification will have a larger error. Therefore, the coupling dynamics simulation of rigid and flexible bodies is closer to the real situation [26-29]. Adams/Flex module in Adams software has two ways to acquire flexible members, one of which is to acquire the modal of components by means of finite element software. We use Ansys software to discretize the finite element, extract the modal of the flexible member, and convert the result into a modal neutral file that Adams software can recognize and then import it into Adams software for dynamic analysis.

When the finite element is discretized for the beam, the flexible beam is composed of I-beam + guide + rack, and the elongated structure and fine-tooth structure increase the complexity of the grid. The quality of the grid drawn by the Ansys software automatic drawing grid function is difficult to meet the requirements. In order to ensure the stability and accuracy of the results, HyperMesh software should be used to mesh complex three-dimensional entities. In order to ensure the accuracy and speed of calculation, all meshes are hexahedral meshes, as shown in Figure 10.

Import the partitioned grid into Ansys software, and remember the node number of quality unit. The flexible body is placed in the original position of the original SolidWorks software inertial coordinate system, and constraints and forces are exerted between the flexible body and the parts. By setting the node of mass element on the end face of each beam as the mark point of Adams, it is connected with other rigid body parts. 


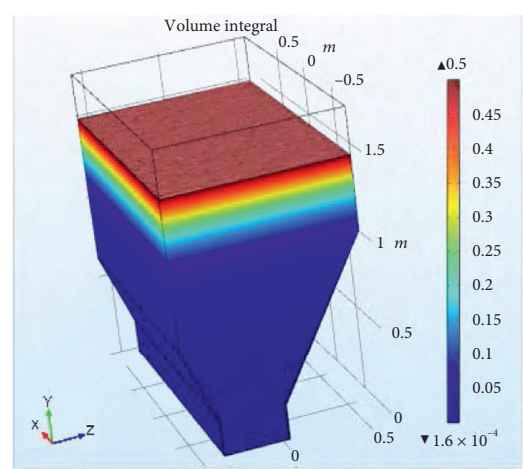

(a)

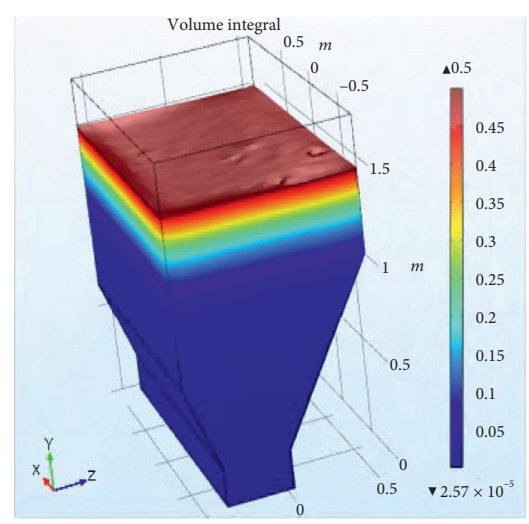

(d)

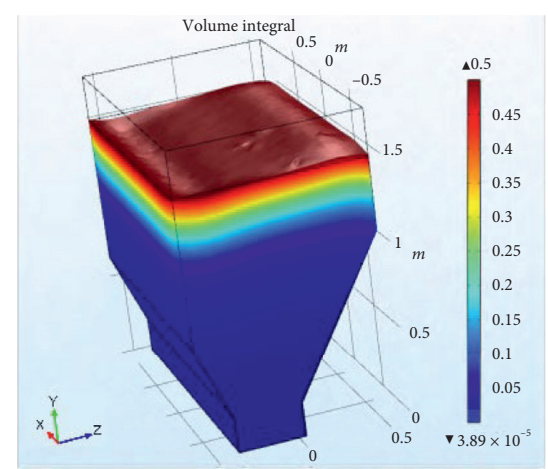

(b)

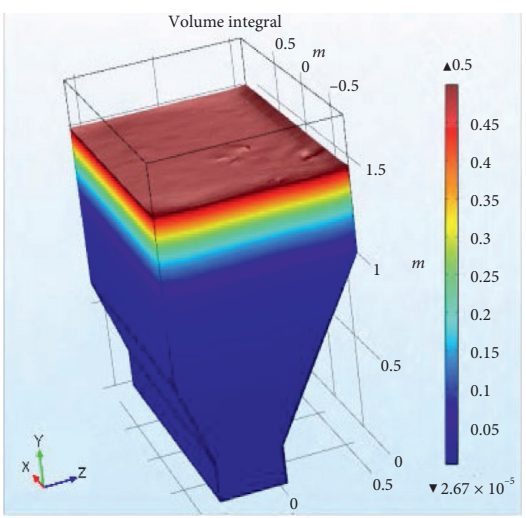

(e)

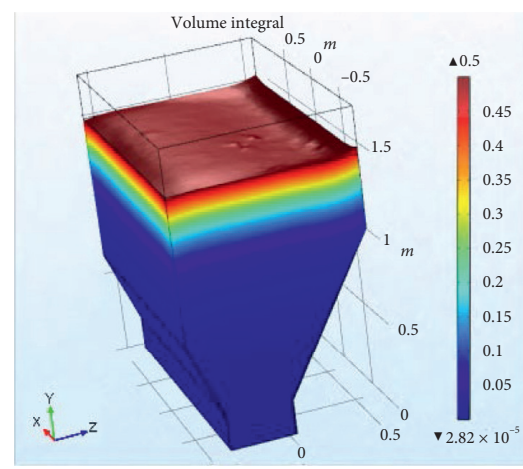

(c)

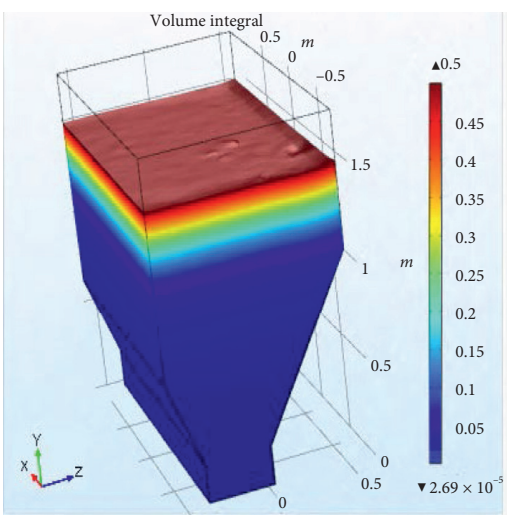

(f)

Figure 7: Simulated changes in fluid motion. (a) $t=0 \mathrm{~s}$. (b) $t=0.5 \mathrm{~s}$. (c) $t=1.5 \mathrm{~s}$. (d) $t=2.5 \mathrm{~s}$. (e) $t=3.5 \mathrm{~s}$. (f) $t=4 \mathrm{~s}$.

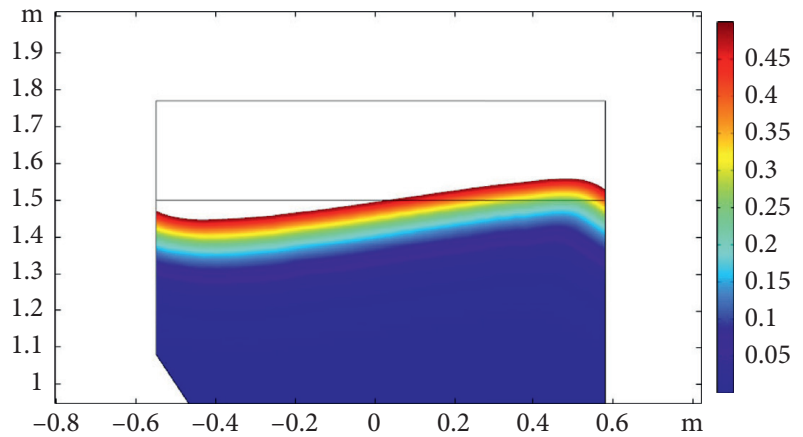

Figure 8: Peak map.

In addition, materials need to be set in Ansys software: material: Q235, elastic modulus: 212E+009 Pa, Poisson's ratio: 0.28 , density: $7850 \mathrm{~kg} / \mathrm{m}^{3}$, solid unit: solid 185 highorder unit, and mass unit: mass21.

Afterwards, components and motion pairs are set up in Adams software: The dispersing device is the integral part 1; the frame of the trolley is the integral part 2; the four wheels of the trolley are parts 3-6; the frame of the cart is divided into two flexible beams and two supporting steel beams, which are parts 7-9; the four wheels of the cart are parts 10-13; the three servo motors are parts 15-17; the three motor gear shafts are parts 18-20; and the frame is part 21 . Standard gravity acceleration is added to each part. At the

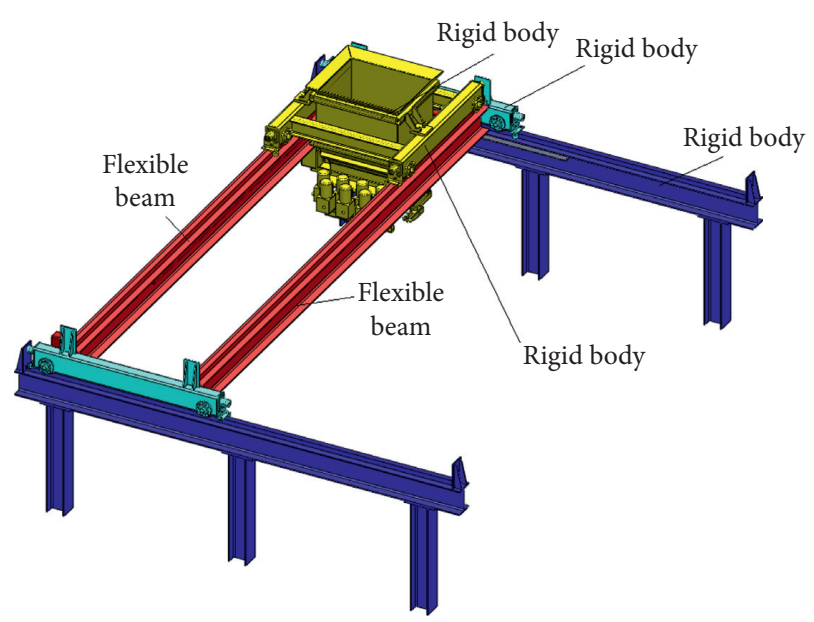

FIgURE 9: Rigid-flexible coupling model of the concrete spreader.

same time, the wheel and guide rail are restrained by contact body. Gear shaft and frame adopt rotating pair. Gears and racks are constrained by contact bodies. The rest of the stationary components and the ground are fixed. Figure 11 is the Adams model of heavy-duty concrete spreader.

After the model is created, as well as in the process of creating the model, the motion simulation of the model can be carried out, and the performance test can be carried out to verify the correctness of the design scheme. In the simulation process of the model, the motion characteristics of the 


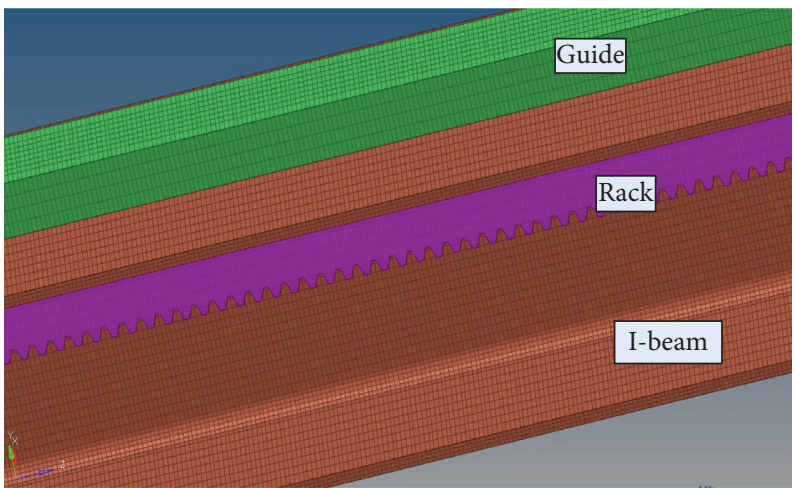

Figure 10: 3D HyperMesh mesh screenshot of beam.

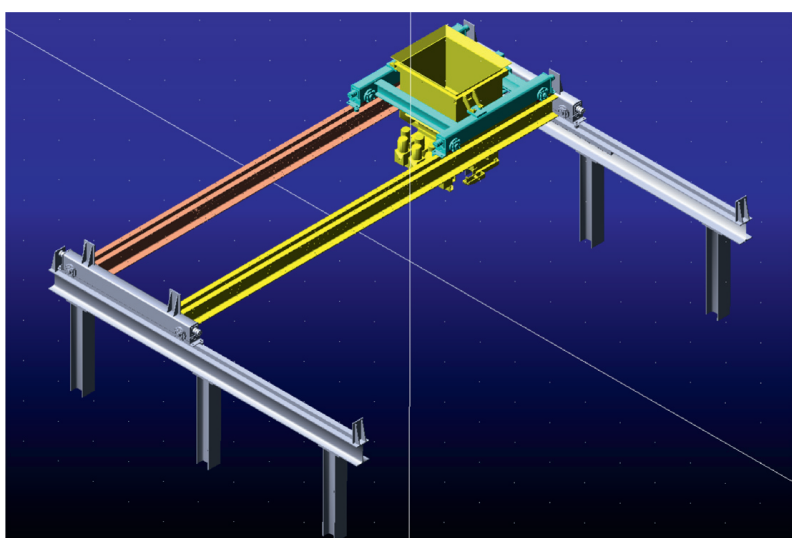

FIGURE 11: Adams model of heavy-duty concrete spreader.

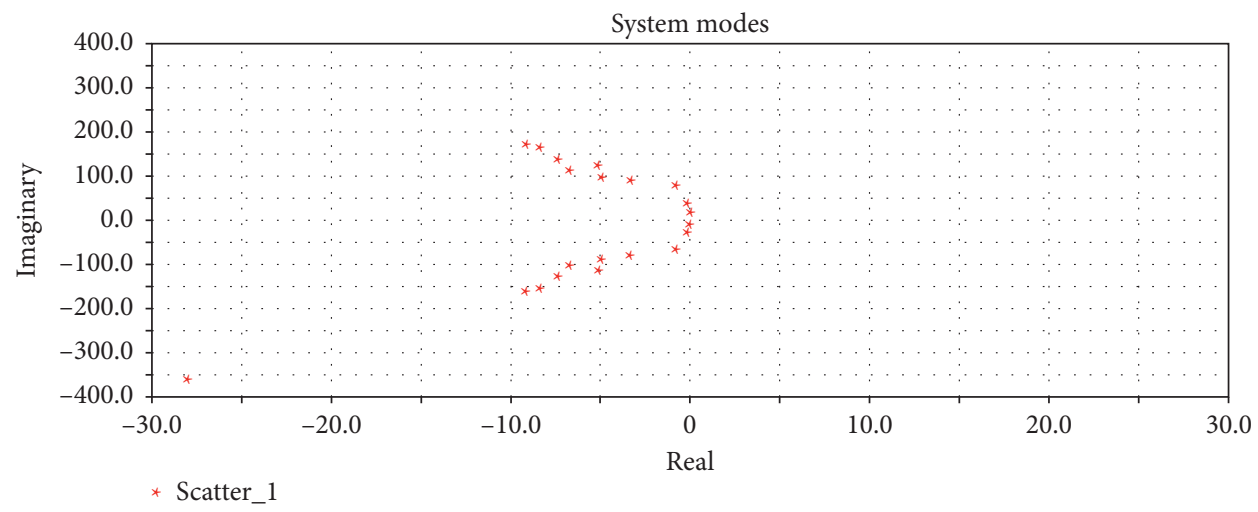

Figure 12: The modal distribution of heavy-duty concrete spreader.
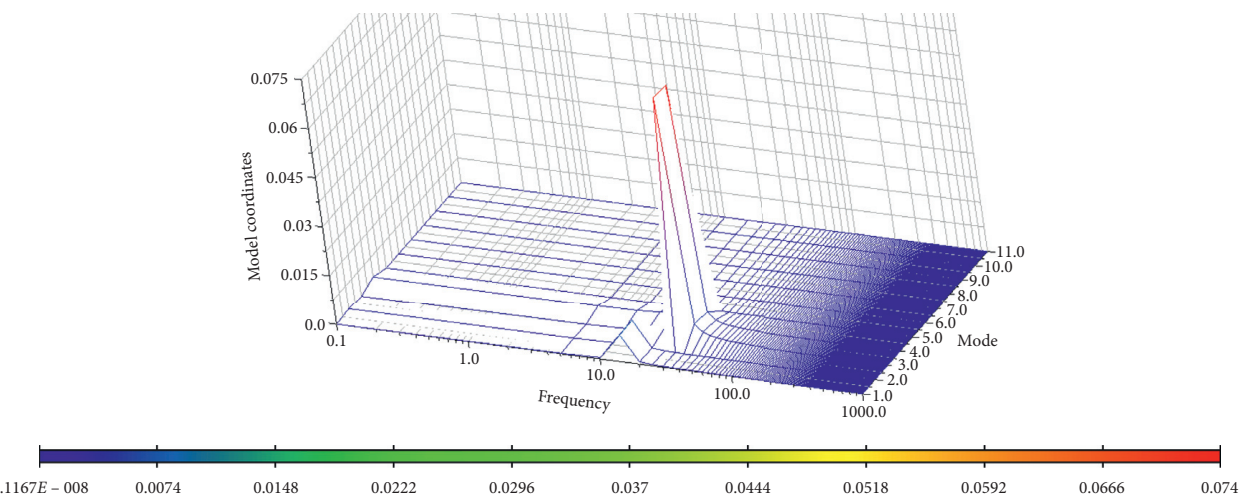

FiguRE 13: The frequency response of the centroid of the dispersing device. 


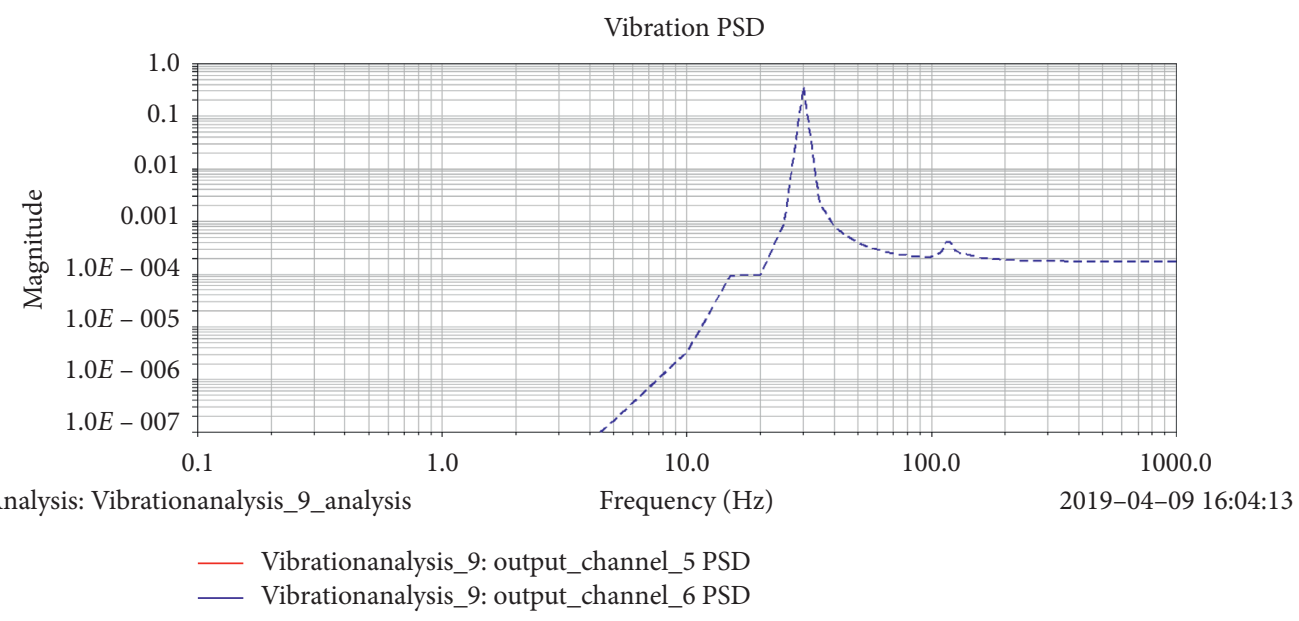

Figure 14: The vertical acceleration power spectral density curve of the centroid of the spreader.

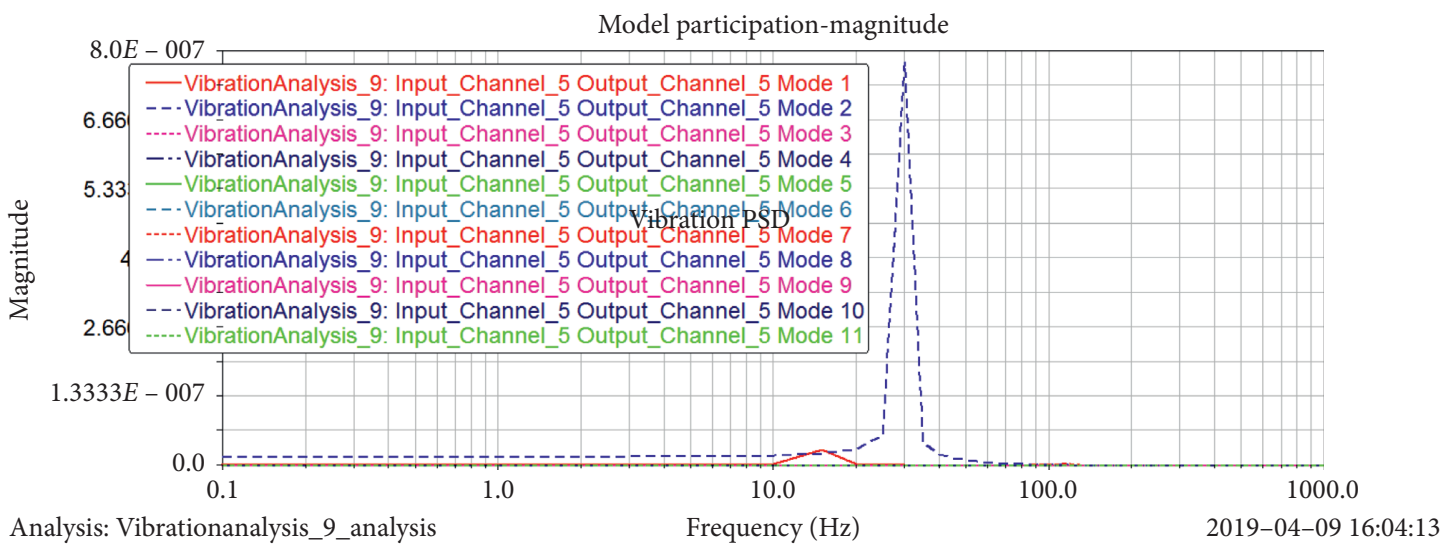

FIGURE 15: The modal participation factor curve.

model, such as distance, speed, and other motion parameters, are calculated automatically. In postprocessing environment, this information can be clearly reproduced by using charts. In addition, the measurement target can be customized. In the simulation process, the stress and deformation of each point of the flexible beam can be easily obtained. Driving force of motor can be calculated and expressed by curve; and the measuring curve shows the change of the measuring object intuitively. In order to compare different design schemes, design points and variables can be defined to parameterize the model. The optimal design variables can be obtained by iterative simulation with different values of design variables.

In addition, vibration module of Adams can also be used for vibration simulation analysis, and the natural frequencies, main modes, and frequency response curves of the system can be obtained [30-34]. For example, Figure 12 shows the modal distribution of heavy-duty concrete spreader, Figure 13 shows the frequency response of the centroid of the dispersing device, Figure 14 shows the vertical acceleration power spectral density curve of the centroid of the spreader, and Figure 15 shows the modal participation factor curve. The virtual detection vibration is convenient to replace the complicated detection process in the actual vibration research, thereby avoiding the drawback that the actual detection can only be carried out in the later stage of the design and is expensive. Therefore, CAE cosimulation can shorten the development cycle and reduce the design cost.

\section{Conclusions}

In this paper, the mathematical method of the liquid-solid coupling dynamics model of heavy-duty concrete spreader is established by using the Euler-Lagrange equation. On this basis, the modeling method from liquid-solid coupling dynamics model to liquid-solid rigid-flexible coupling model is proposed. The calculation and derivation process of dynamic model is given in detail, which provides a new idea for mathematical modeling of similar equipment.

CAE cosimulation method for heavy-duty concrete spreader based on liquid-solid rigid-flexible coupling is proposed. Compared with mathematical modeling methods, CAE cosimulation method is simple to model and provides 
an effective reference for studying the structural design and performance optimization of other similar mechanical products.

In the CAE cosimulation process of heavy-duty concrete spreader, the vibration module of Adams can be used to analyze the vibration, and the natural frequency, modal main vibration mode, and frequency response curve of the system are obtained, shortening the development cycle and reducing the design cost.

\section{Data Availability}

No data were used to support this study.

\section{Conflicts of Interest}

The authors declare that they have no conflicts of interest.

\section{Acknowledgments}

This research was supported by the National Key Research and Development Projects (2017YFC0704003)

\section{References}

[1] D. F. Zou, P. Zhou, J. Sun et al., "Introduction of technique of large and intelligent production line for PC slabs and set of equipment," China Concrete and Cement Products, vol. 4, pp. 37-41, 2017.

[2] H. Sun, J. Sun, Y. H. Wu et al., "Introduction to large intelligent PC component automation production line," China Concrete and Cement Products, vol. 3, pp. 35-38, 2015.

[3] K. Zhang, Z. Li, W. Yu et al., "Energy consumption analysis of the scattering rod of concrete distributor," Journal of Shenyang Jianzhu University, vol. 11, pp. 1121-1127, 2020.

[4] K. Zhang, T. Yu, W. Yu et al., "Calibration of concrete discrete element parameters based on J KR bond model," Concrete, vol. 8, pp. 46-50, 2020.

[5] X. G. Hu and R. K. Sui, "Dynamic simulation analysis of gantry crane based on virtual prototype technology," Science \& Technology Review, vol. 2, pp. 46-49, 2010.

[6] M. A. Ahmad and Z. Mohamed, "Hybrid fuzzy logic control with input shaping for input tracking and sway suppression of a gantry crane system," American Journal of Engineering and Applied Science, vol. 6, pp. 45-51, 2009.

[7] H. J. Sun, Simulation and Analysis of Stability for Crawler Crane Based on ADAMS, Northeastern University, Shenyang, China, 2010.

[8] P. Wang, Y. C. Fang, and J. L. Xiang, "Dynamics analysis and modeling of ship-mounted boom crane," Journal of $\mathrm{Me}$ chanical Engineering, vol. 47, no. 20, pp. 34-40, 2011.

[9] W. Rulka and E. Pankiewicz, "MBS approach to generate equations of motions for HiL-simulations in vehicle dynamics," Multibody System Dynamics, vol. 14, no. 3-4, pp. 367-386, 2005.

[10] F. Naets, G. H. K. Heirman, W. Desmet, and T. Tamarozzi, "Real-time flexible multibody simulation with global modal parameterization," Multibody System Dynamics, vol. 27, no. 3, pp. 267-284, 2012.

[11] G. L. Xiong, P. H. Li, and X. D. Cai, "Virtual prototyping technology," Journal of System Simulation, vol. 1, pp. 146-117, 2001.
[12] B. H. Li and X. D. Cai, "Virtual prototyping engineering for complex products," Computer Integrated Manufacturing Systems, vol. 9, pp. 678-681, 2002.

[13] X. Zhang and E. R. Mao, "Research and development of mechanical system virtual prototype technology," Journal of China Agricultural University, vol. 4, pp. 94-95, 2001.

[14] S. Zhang, D. Zou, W. Yu et al., "Optimization design of walking beam of precast component concrete distributing machine," Construction mechanization, vol. 10, pp. 37-39, 2019.

[15] Y. Jiang, "Research and application of numerical control production line technology for small concrete precast parts," Logistics Engineering and Management, vol. 37, no. 8, pp. 116-117, 2015.

[16] R. W. Xi, "A brief discussion on the common problems of the adjustment of concrete distributing machine," New Technology \& New Products of China, vol. 7, pp. 43-44, 2017.

[17] J. W. Fernandez, P. W. Cleary, and W. McBride, "Effect of screw design on hopper drawdown of spherical particles in a horizontal screw feeder," Chemical Engineering Science, vol. 66, no. 22, pp. 5585-5601, 2011.

[18] Y. G. Dong, W. A. Xie, Y. X. Liang et al., "Upgrade and replacement of distributor in prefabricated component production line," Innovation and Application of Science and Technology, vol. 11, pp. 125-126, 2016.

[19] D. Zou, S. Wen, W. Yu et al., "Driving power calculation method for screwcutting device of multi-screw concrete distributing machine," Construction Mechanization, vol. 7, pp. 17-20, 2019.

[20] J. A. C. Ambrósio and J. P. C. Gonçalves, "Complex flexible multibody systems with application to vehicle dynamics," Multibody System Dynamics, vol. 6, no. 2, pp. 163-182, 2001.

[21] E. Lund, H. MØller, J. A. C. Ambrosio, and J. Goncalves, "Simulation of fluid loaded flexible multibody systems," Multibody System Dynamics, vol. 13, pp. 113-128, 2005.

[22] K. F. Zhao, G. G. Cheng, Z. Q. Zhang et al., "Numerical analysis of solidification process of molten silicon in horizontal pipeline," Hot Working Technology, vol. 9, pp. 93-96, 2017.

[23] J. Xu, Z. P. Lv, and J. R. Tang, "Structural strength analysis of automotive fuel tank based on ABAQUS," Equipment Manufacturing Technology, vol. 9, pp. 76-80, 2014.

[24] L. Liu, "Development trend of plastic fuel tank for automobile," Light Vehicle Technology, vol. 2, pp. 22-25, 2016.

[25] L. D. Zhu, C. X. Zhu, and C. Su, "Investigation on simulation and experiment of excitation characteristics of turn milling center," Information an International Interdisciplinary Journal, vol. 14, pp. 3875-3880, 2011.

[26] C. Zhu, J. Wang, Z. Chen, and B. Liu, "Dynamic characteristic parameters identification analysis of a parallel manipulator with flexible links," Journal of Mechanical Science and Technology, vol. 28, no. 12, pp. 4833-4840, 2014.

[27] M. Greco and H. B. Coda, "Positional FEM formulation for flexible multi-body dynamic analysis," Journal of Sound and Vibration, vol. 290, no. 3-5, pp. 1141-1174, 2006.

[28] V. O. Gamarra-Rosado and E. A. O. Yuhara, "Dynamic modeling and simulation of a flexible robotic manipulator," Robotics, vol. 17, pp. 523-528, 1999.

[29] W. Chen, "Dynamic modeling of multi-link flexible robotic manipulators," Computers \& Structures, vol. 79, no. 2, pp. 183-195, 2001.

[30] Y. B. Zhang and H. D. Mao, "Vibration analysis of vertical roller mill grinding device based on ADAMS/vibration," Coal Mine Machinery, vol. 4, pp. 37-39, 2017. 
[31] X. L. Chen, "Vibration analysis of asymmetric block based on ANSYS and ADAMS simulation," Journal of Dalian University of Technology, vol. 6, pp. 13-16, 2016.

[32] S. G. Hou, "Vibration analysis based on ADAMS," Internal Combustion Engine \& Parts, vol. 2, pp. 65-66, 2018.

[33] P. Y. Shi, N. Liu, and S. F. Shi, "Modeling of transportation packaging flexible body and analysis of ADAMS rigid-flexible coupling transportation vibration," Automotive Application Technology, vol. 5, pp. 57-58, 2013.

[34] C. X. Zhu, J. M. Luo, D. Wang, and B. Cai, "Research on modal parameters identification of parallel manipulator with flexible multibody system," Research Journal of Applied Sciences Engineering and Technology, vol. 5, pp. 2974-2979, 2013. 